International Mathematical Forum, Vol. 9, 2014, no. 1, 13 - 18

HIKARI Ltd, www.m-hikari.com

http://dx.doi.org/10.12988/imf.2014.311221

\title{
Global Attractor for \\ a 3D Reaction-Diffusion Equation
}

\author{
Xiaosong Wang ${ }^{1}$, Hongjun Wang $^{2}$ and Lingrui Zhang ${ }^{3}$ \\ ${ }^{1}$ College of Information Science \\ ${ }^{1}$ Henan University of Technology, Zhengzhou, 450001, P.R. China \\ ${ }^{2}$ College of Mathematics and Information Science \\ ${ }^{3}$ College of Education and Teacher Development \\ ${ }^{2,3}$ Henan Normal University, Xinxiang, 453007, P.R. China \\ wxs0219@126.com (X. Wang) \\ hsdwhj@163.com (H. Wang) \\ zhanglingrui@126.com (L. Zhang)
}

Copyright (c) 2014 Xiaosong Wang, Hongjun Wang and Lingrui Zhang. This is an open access article distributed under the Creative Commons Attribution License, which permits unrestricted use, distribution, and reproduction in any medium, provided the original work is properly cited.

\begin{abstract}
In this paper, we consider the long time behavior of a 3D reactiondiffusion equation and obtain the existence of global attractor for a autonomous reaction-diffusion system by establishing the asymptotical smoothness for the semigroup by decomposition method which introduced by Zelik (CPAA 2004).
\end{abstract}

Mathematics Subject Classifications: 35B40, 35Q99, 80A22

Keywords: reaction diffusion equations, decomposition method, global attractor

\section{Introduction}

Let $\Omega \subset R^{3}$ be a bounded domain with sufficiently smooth boundary $\partial \Omega$, we consider the non-autonomous $3 \mathrm{D}$ reaction-diffusion equation:

$$
u_{t}-\Delta u+\lambda u+u^{3}=f(x), \quad x \in \Omega, \quad t \in R^{+},
$$




$$
\begin{aligned}
& \left.u(t, x)\right|_{\partial \Omega}=0, \\
& u(0, x)=u_{0}(x), \tau \geq 0 .
\end{aligned}
$$

Here $u=u(t, x)=\left(u_{1}(t, x), u_{2}(t, x), u_{3}(t, x)\right)$ is the velocity vector field, $p$ is the pressure, $\nu>0$ is the kinematic viscosity, the length scale $\alpha$ is a characterizing parameter of the elasticity of the fluid.

Since the global wellposedness and long time behavior of reaction-diffusion equation pay an important role in explain the stability of reaction diffusion phenomena in physics, biology, chemistry and engineering science, the reactiondiffusion equation has become a hot topic in pure and applied mathematical research. For the classical result of reaction-diffusion equation, such as the global existence of solutions in $L^{2}(\Omega)$ and $H_{0}^{1}(\Omega)$, long-time behavior for autonomous system (such as global attractor, Hausdorff dimension of attractor), we can refer to [9], [4], [3]. Later, Chepyzhov and Vishik [1] gave a new method which is well suited to investigating equations arising in mathematical physics without unique solvability, the theory of trajectory attractor has developed and used to reaction-diffusion equation. Zhong, Yang and Sun [10] represented a general theory of norm-to-weak semigroup and applied to reaction-diffusion equation which obtained the existence of global attractor. Song and Zhong [7] investigate the case of external force function with less regularity (such as the external force is translation bounded but not translation compact or belong to norm functions class) and derived the uniform attractor in $L^{p}(\Omega)$ for the reaction-diffusion equation.

In this paper, we consider the long time behavior of a 3D reaction-diffusion equation and obtain the existence of global attractor for a autonomous reactiondiffusion system by establishing the asymptotical smoothness for the semigroup by decomposition method which introduced by Zelik [11] (CPAA 2004).

\section{Global existence of solutions and attractors for the reactive-diffusion equation}

In the sequel, we set $H=L^{2}(\Omega), V=\left\{u \in H^{1}(\Omega) \cap H,\left.u\right|_{\partial \Omega}=0\right\}$, $W=H^{2}(\Omega) \cap V$. then we state the existence of global solution to problem (1)-(3).

We denote the family of Hilbert spaces $D\left(A^{\frac{s}{2}}\right)(s \in R)$ with the usual product and norm as $\langle\cdot, \cdot\rangle_{D\left(A^{\frac{s}{2}}\right)}=\left\langle A^{\frac{s}{2}} \cdot, A^{\frac{s}{2}} \cdot\right\rangle$ and $\|\cdot\|_{D\left(A^{\frac{s}{2}}\right)}=\left\|A^{\frac{s}{2}} \cdot\right\|$ respectively. Obviously, we have $D\left(A^{\frac{s}{2}}\right) \hookrightarrow D\left(A^{\frac{r}{2}}\right)$ for any $s>r$. Especially we denote $\mathcal{H}_{s}=D\left(A^{\frac{s+1}{2}}\right)$ with the norm $\|\cdot\|_{\mathcal{H}_{s}}=\|\cdot\|_{1+s}$.

By the standard Faedo-Galerkin approximation method, we can prove the existence of strong solutions to (1)-(3) as the following theorem. 
Theorem 2.1 (1) Let $f \in V^{\prime}$ and $u_{0} \in H$, then there exists a unique strong solution of problem (1)-(3) such that

$$
u \in H^{1}(0, T ; H) \cap L^{2}(0, T ; W),
$$

for all $T>0$.

(2) Assume $f \in V^{\prime}$ and $u_{0} \in V$, then the system (1)-(3) possess a unique strong solution $u(x, t)$ satisfying

$$
u \in C^{0}\left(R^{+} ; V\right) \cap L^{2}\left(R^{+} ; W\right), \quad u_{t} \in L^{2}\left(R^{+} ; H\right) .
$$

Theorem 2.2 For any $f \in V^{\prime}, u_{0} \in H$, the semigroup $\{S(t)\}, t \geq \tau \in R^{+}$ generated by the system (1)-(3) has a global attractor $\mathcal{A}$ which is invariant, compact in $H$ and attracts all bounded subsets of $H$ in the topology of $H$.

The proof can divide into two steps, the first is to prove the existence of absorbing set, next we shall use decomposition method to prove the asymptotical smoothness for the semigroup.

Since the injection $H \hookrightarrow V^{\prime}$ is dense, then for any $f \in V^{\prime}$, we can find a function $f^{\varepsilon} \in H$ depending on $f$ and $\varepsilon$ such that

$$
\left\|f-f^{\varepsilon}\right\| \leq \varepsilon
$$

Using the similar technique to Zelik [11], we decompose the semigroup into $S(t) u_{0}=S_{v}(t) u_{0}+S_{w}(t) u_{0}$ with $S_{v}(t) u_{0}=v(t)$ and $S_{w}(t) u_{0}=w(t)$ that satisfy the following equations respectively

$$
\begin{aligned}
& v_{t}+A v_{t}+\lambda v=f-f^{\varepsilon}, \\
& v(x, 0)=u_{0}(x)
\end{aligned}
$$

and

$$
\begin{aligned}
& w_{t}+A w_{t}+\lambda w+u^{3}=f^{\varepsilon}, \\
& w(x, 0)=0, \quad x \in \Omega .
\end{aligned}
$$

\section{Existence of global absorbing set}

Lemma 3.1 Let the external force $f \in V^{\prime}, u_{\tau} \in H$, then the semigroup has a bounded global absorbing set $B_{0}$ in $H$, where

$$
B_{0}=\left\{u \in H:\|u\|_{H} \leq \rho\right\}
$$

is a bounded set in $H$. 
Proof. Taking inner product of (1) with $\mathrm{u}$, and integrating by parts over $\Omega$, we have

$$
\frac{1}{2} \frac{d}{d t}\|u\|^{2}+\|\nabla u\|^{2}+\int_{\Omega}\left(u^{3}+\lambda u\right) \cdot u d x=\int_{\Omega} f(x) \cdot u d x
$$

where $\|\cdot\|$ denotes the $H$ norm, $\|\nabla(\cdot)\|$ is equivalent to the $V$ norm.

The Poincaré inequality implies $\|\nabla u\|^{2} \geq \frac{1}{2}\|\nabla u\|^{2}+\frac{\lambda}{2}\|u\|^{2}$. Hence, by the Cauchy inequality, we derive

$$
\begin{gathered}
\frac{1}{2} \frac{d}{d t}\|u\|^{2}+\frac{1}{2}\|\nabla u\|^{2}+\frac{\lambda}{2}\|u\|^{2}+\lambda\|u\|^{2}+\|u\|_{L^{4}}^{4} \\
\leq \frac{1}{\lambda}\|f(x)\|_{V^{\prime}}^{2}+\lambda\|u\|^{2}
\end{gathered}
$$

i.e.,

$$
\frac{d}{d t}\|u\|^{2}+\lambda\|u\|^{2} \leq \frac{2}{\lambda}\|f(x)\|_{V^{\prime}}^{2}
$$

By the Gronwall inequality, we have

$$
\|u(x, t)\|^{2} \leq e^{-\frac{\lambda t}{2}}\left\|u_{0}\right\|^{2}+\frac{2}{\lambda^{2}}\|f(x)\|_{V^{\prime}}^{2}
$$

Choosing $\rho^{2}=e^{-\frac{\lambda t}{2}}\left\|u_{0}\right\|^{2}+\frac{2}{\lambda^{2}}\|f(x)\|_{V^{\prime}}^{2}$ such that $B_{0}=\left\{u:\|u\|_{H}^{2} \leq \rho^{2}\right\}$, i.e., $B_{0}$ is the global absorbing ball for the semigroup $\{S(t)\}_{t \geq 0}$ in $H$.

\section{Asymptotic smoothness for the semigroup}

Theorem 4.1 For any $f \in V^{\prime}$, the semigroup $\{S(t), t \geq 0\}$ generated by the system (1)-(3) is asymptotical smoothness in $H$.

Proof. We shall use decomposition method to achieve the result in the following lemmas.

Lemma 4.2 For the system (7)-(8), there exists a constant $\varepsilon=\varepsilon(f, \delta)$ such that the solution of (7)-(8) satisfies

$$
\left\|S_{v}(t) u_{0}\right\|_{H}^{2}=\|v(x, t)\|_{H}^{2} \leq Q\left(\left\|u_{0}\right\|_{H}\right) e^{-\frac{\lambda t}{2}}+\delta
$$

for any $t \geq 0$, where $Q(\cdot)$ is a positive increasing function on $[0,+\infty)$.

Proof. Multiplying (7) with $v(t)$, integrating over $\Omega$, we deduce

$$
\frac{1}{2} \frac{d}{d t}\|v\|^{2}+\frac{1}{2}\|\nabla v\|^{2}+\frac{\lambda}{2}\|v\|^{2}+\lambda\|v\|^{2} \leq \frac{1}{\lambda}\left\|f-f^{\varepsilon}\right\|_{V^{\prime}}^{2}+\lambda\|v\|^{2},
$$


which means

$$
\frac{d}{d t}\|v\|^{2}+\lambda\|v\|^{2} \leq \frac{2}{\lambda}\left\|f-f^{\varepsilon}\right\|_{V^{\prime}}^{2}
$$

applying the Gronwall lemma, we have

$$
\|v\|_{H}^{2} \leq Q\left(\left\|u_{0}\right\|_{H}\right) e^{-\frac{\lambda t}{2}}+\frac{2}{\lambda^{2}}\left\|f-f^{\varepsilon}\right\|_{V^{\prime}}^{2} .
$$

Choosing $\varepsilon^{2} \leq 2 \lambda^{2} \delta$, we complete the proof.

Lemma 4.3 For any time $T>0$ and constant $\varepsilon>0$, there exists a nonnegative constant $M>0$ depending on the constants $T, f^{\varepsilon},\left\|u_{0}\right\|_{H}$ such that the solution to the system (9)-(10) has the property

$$
\left\|S_{w}(t) u_{0}\right\|_{\mathcal{H}_{s}}^{2}=\|w(t)\|_{\mathcal{H}_{s}}^{2} \leq M
$$

for all $0<s \leq 1$.

Proof. Taking inner product of (9) with $A^{s} w(t)$, we obtain

$$
\frac{1}{2} \frac{d}{d t} \int_{\Omega}\left|A^{\frac{s}{2}} w\right|^{2} d x+\int_{\Omega}\left|A^{\frac{s+1}{2}} w\right|^{2} d x+\lambda \int_{\Omega}\left|A^{\frac{s}{2}} w\right|^{2} d x+\left(u^{3}, A^{s} w\right)=\left\langle f^{\varepsilon}, w\right\rangle .
$$

Since $0<s \leq 1$, by the Cauchy inequality, we have

$$
\left\langle f^{\varepsilon}, w\right\rangle \leq C\left\|f^{\varepsilon}\right\|\left\|A^{\frac{s+1}{2}} w\right\| \leq \frac{C_{1}}{2}\left\|f^{\varepsilon}\right\|^{2}+\frac{C_{2}}{2} \int_{\Omega}\left|A^{\frac{s+1}{2}} w\right|^{2} d x
$$

and

$$
\left|\left(u^{3}, A^{s} w\right)\right| \leq C\left\|u^{3}\right\|\left\|A^{\frac{s+1}{2}} w\right\| \leq \frac{C_{3}}{2}\|u\|_{L^{6}}^{2}+\frac{C_{4}}{2}\left\|A^{\frac{s+1}{2}} w\right\|^{2} .
$$

Since $H^{1} \hookrightarrow L^{6}$, then we can choose $s=1$ and appropriate constants $C_{i}(i=$ $1,2,3,4)$ such that

$$
\begin{aligned}
& \frac{1}{2} \frac{d}{d t} \int_{\Omega}\left|A^{\frac{1}{2}} w\right|^{2} d x+\int_{\Omega}|A w|^{2} d x+\lambda \int_{\Omega}\left|A^{\frac{1}{2}} w\right|^{2} d x \\
& \leq \frac{C_{1}}{2}\left\|f^{\varepsilon}\right\|^{2}+\int_{\Omega}|A w|^{2} d x+C_{0} \lambda \int_{\Omega}\left|A^{\frac{1}{2}} w\right|^{2} d x,
\end{aligned}
$$

here $C_{0}>1$. This means

$$
\frac{d}{d t} \int_{\Omega}\left|A^{\frac{1}{2}} w\right|^{2} d x \leq C_{5}\left(\frac{C_{1}}{2}\left\|f^{\varepsilon}\right\|^{2}+C(\lambda) \int_{\Omega}\left|A^{\frac{1}{2}} w\right|^{2} d x\right)
$$

Using the Gronwall lemma, we conclude that

$$
\left\|A^{\frac{s}{2}}\right\|^{2} \leq C\left\|A^{\frac{1}{2}}\right\| \leq C^{\prime} e^{C_{5} t}
$$

for all $t \geq 0$.

From the compact embedding $\mathcal{H}_{s} \hookrightarrow V \hookrightarrow H$ is compact, we can deduce the asymptotical smoothness for the semigroup. This complete the proof of the lemma.

Acknowledgments Hongjun Wang was supported by the Nature Science Fund from the Department of Science and Technology in Henan Province (No. 122300410414) and (No. 132300410432). 


\section{References}

[1] V. V. Chepyzhov and M. I. Vishik, Trajectory attractors for reactiondiffusion systems, topological methods in nonlinear analysis, J. Juliusz Schauder Center, 7(1996), 49-76.

[2] I. D. Chueshov, Introduction to the Theory of Infinite-Dimensional Dissipative Systems, Acta Scientific Publishing House, Kharkiv, 2002.

[3] M. Efendiev and S. Zelik, The attractor of a nonlinear reaction-diffusion system in an bounded domain, Comm. Pure Appl. Math., 54(2001), 625688 .

[4] M. Marion, Attractors for reaction-diffusion equations: Existence and estimate of their dimension, Appl. Anal., 25(1987), 101-147.

[5] J. C. Robinson, Infinite Dimensional Dynamical Systems an Introduction to Dissipative Parabolic PDEs and the Theory of Global Attractors, Cambridge University Press, 2001.

[6] G. R. Sell and Y. You, Dynamics of Evolutionary Equations, Springer, New York, 2002.

[7] H. Song and C. Zhong, Attractors of non-autonomous reaction-diffusion equations in $L^{p}$, Nonlinear Analysis, 68(2008), 1890-1897.

[8] C. Sun and M. Yang, Dynamics of the non-calssical diffusion equations, Asymptotic Analysis, 59(2008), 51-81.

[9] R. Temam, Infinite Dimensional Dynamical System in Mechanics and Physics, second ed., Springer, New York, 1997.

[10] C. Zhong, M. Yang and C. Sun, The existence of global attractors for the norm-to-weak continuous semigroup and its application to the nonlinear reaction-diffusion equations, J. Differential Equations, 223(2)(2006), 367-399.

[11] S. Zelik, Asymptotic regularity of solutions of a non-autonomous damped wave equations with a critical growth exponent, Comm. Pure Appl. Anal., 3(4)(2004), 921-934.

\section{Received: November 25, 2013}

